\title{
Diversity 4.0 in the cardiovascular health-care workforce
}

\author{
Renate B. Schnabel(10 ${ }^{1,2}$ and Emelia J. Benjamin (10 $3,4,5 \times$
}

The disparities amplified by the COVID-19 pandemic present disturbing evidence that we are far from cardiovascular health-care equity. Individuals, leaders and institutions must prioritize research, policies and structures to advance diversity, equity, inclusion and belonging - Diversity 4.0, a justice imperative, essential to advancing workforce excellence and cardiovascular health.

A decade ago, Marc Nivet wrote for the Association of American Medical Colleges about the three phases of diversity ${ }^{1}$. Diversity 1.0 had an isolated focus on recruitment, laws and compliance, sometimes proffered (inaccurately) as potentially being at the 'expense of excellence'. Diversity 2.0 emerged in the 1980s and emphasized the promotion of racial and ethnic student, faculty and staff 'minority' equity but remained siloed from the core academic mission in 'minority' affairs offices. In the past two decades, Diversity 3.0 recognized that diversity and a culture of inclusion were strategic imperatives to achieve excellence and health equity. However, with the inequities laid bare by the coronavirus disease 2019 (COVID-19) pandemic, and the Black Lives Matter and \#MeToo movements, individuals and institutions in academic health sciences must come to the reckoning; incremental efforts will not achieve the transformation that is imperative for our times. We call upon academic health sciences to engage in Diversity 4.0 (FIG. 1). Individuals and institutions must actively engage in transformative personal and structural anti-racist, anti-sexist and anti-classist work to promote diversity, equity, inclusion and belonging (DEIB). A DEIB commitment will harness the innovation of all voices in academia to collectively address profound and persistent global structural health-care and workforce inequities.

\section{The excellence imperative}

Diversity in our societies has grown but is not reflected in the cardiovascular health-care workforce ${ }^{2}$. Diversity comprises many facets including gender, race/ethnicity, socioeconomic status, LGBTQ+, national origin and religion, among many other identities. Diversity and equity in the health-care workforce have been shown to benefit patients and increase access to health care for underserved populations. Data also support that diverse organizations perform better and show more innovative thinking and problem-solving. In science, diverse teams in terms of race, ethnicity and gender on average publish in higher-impact journals than non-diverse teams ${ }^{3}$; ethnically diverse teams on average had $10 \%$ more impact for papers and almost 50\% higher impact for authors ${ }^{3}$.

\section{Current state: gender and race/ethnicity}

The underrepresentation of women in cardiology despite more than two decades of recognition and efforts to address disparities is a prime example. In Australia, women constitute about $25 \%$ of cardiology trainees, $15 \%$ of cardiologists and $4.8 \%$ of the interventional cardiologist workforce ${ }^{4}$. The numbers are strikingly similar in the UK and the USA ${ }^{5}$. Over the past decade, the number of female cardiology trainees has hardly increased. Substantive socioeconomic disparities between female and male cardiologists persist; accounting for factors tied to compensation, women on average still earn only $\sim 92 \%$ of their male counterparts, translating to $\sim$ US $\$ 2.5$ million less over a 35-year career ${ }^{6}$. Women are less frequently mentored by men and experience less sponsorship for NIH Mentored Career Development grants. Furthermore, the number of prestigious authorships of women on cardiovascular research articles has remained disproportionally low. First-author roles do not track into later senior authorships as often for women as they do for men. The social media profile, quantified as citations numbers and Twitter followers, does not compare with the publication record equally in women and men $^{7}$. Women represent a minority in cardiovascular leadership in academic institutions and professional societies, at conferences, and as presenters and panelists. Evidence of structural sexism persists, such as a lack of sufficient childcare, adequate family leave policies, designation of endowed chairs and institutional responses to sexual harassment.

Although less well studied, profound inequities also exist for individuals underrepresented in medicine, such as African Americans, Hispanic Americans and Native Americans who constitute $32 \%$ of the US population but only $8 \%$ of practising cardiologists ${ }^{8}$. Whereas some problematic reports have suggested that workforce and health-outcome disparities in individuals underrepresented in medicine are due to intrinsic factors, inequities 


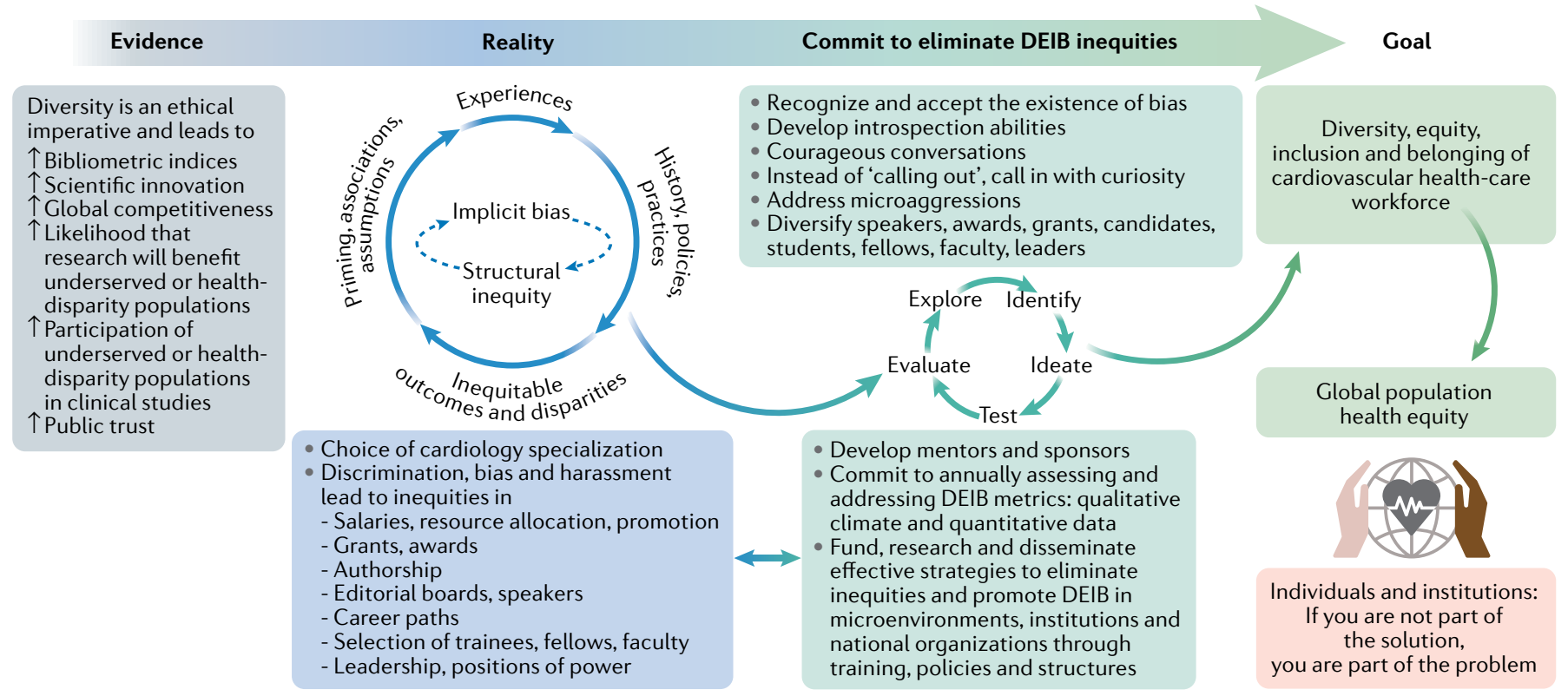

Fig. 1 | Excellence imperative for diversity: individual and institutional responsibility. Examples of available evidence, current reality and steps to be taken to overcome bias and structural inequity to achieve equity in the cardiovascular health-care workforce. DEIB, diversity, equity, inclusion and belonging.

are increasingly recognized to be manifestations of structural racism. In the cardiologist workforce, an example of structural racism is an overreliance on board scores and institutional pedigree for selecting trainees and fellows, which often are proxies for socioeconomic status. Similarly, a robust literature indicates that awards, letters of recommendation and grants advantage men and white individuals in consequential, biased ways.

Progress towards equity has remained poor. Factors contributing to persistent inequities include implicit bias, stereotyping, discrimination, harassment ${ }^{8}$, and structural racism, sexism and classism. In the USA, $>65 \%$ of female cardiologists report having experienced discrimination. Of women reporting discrimination, $96 \%, 37 \%$ and $18 \%$ noted discrimination based on gender, parenting and race, respectively. Of the $23 \%$ of male cardiologists reporting discrimination, the respective numbers were $8 \%, 8 \%$ and $59 \%{ }^{8}$. Over $75 \%$ of US medical students' parents are in the top two quintiles of household income ${ }^{9}$.

Gender, racial, ethnic and class disparities have not been as widely reported outside of the USA. Our conviction is that this absence of literature represents a lack of deep and honest inquiry rather than an absence of discrimination and bias towards individuals underrepresented in medicine in high-income countries (such as individuals of Indigenous, Eastern European, African or Middle Eastern descents). Similarly, the first woman to be president of the ESC, Barbara Casadei, was installed only in 2018.

Bias is a major obstacle to achieving equity. Implicit biases describe the tendencies to make judgements without questioning, which determine our decisions and actions on the basis of priming, associations, assumptions and stereotypes. The difficulty of controlling implicit biases is that they are inherently unconscious and unintentional. A lack of intention is not an excuse given the profound impact of biases, because they impair the creation of an equitable cardiovascular health-care workforce, from the selection of students, trainees, faculty and leaders, to grant success, advancement, promotion, compensation and the designation of leaders.

\section{Diversity 4.0: individual mandate}

Individuals must commit to deeply examining and mitigating the influence of implicit biases on interactions, letters, interviews, selections, nominations and actions. Individuals must commit to acting in anti-racist and anti-sexist allyship, and to mentor and sponsor women and individuals underrepresented in medicine. Evidence-based strategies to mitigate implicit bias and advance DEIB include stereotype replacement, individuating instead of generalizing by identity group, perspective-taking (imagine being a diverse individual) and seeking meaningful collaboration with diverse individuals ${ }^{10}$. Individuals must seek opportunities for mentoring, sponsoring and promoting diverse individuals. The NIH has funded the cluster-randomized Bias Reduction in Internal Medicine trial to test whether a 'breaking the bias habit' approach can effectively reduce bias among faculty. The NIH also provides toolkits for diversity-promoting recruitment search protocols.

\section{Diversity 4.0: organizational mandate}

Leaders, institutions and organizations must measure and annually track qualitative and quantitative DEIB metrics and be held accountable for eliminating bias, discrimination and harassment. Analysing inequities in compensation, resources, access to sponsorship and mentorship, advancement, promotion and leadership of diverse individuals will be crucial. Organizations must have policies and confidential procedures in place wherein individuals experiencing bias, discrimination or harassment can 
seek counsel and remediation without fear of retribution. By generating and analysing institutional and organizational metrics, organizations can identify unsupportive, biased individuals and institutional cultures, engage in 'root cause' analyses and rectify identified inequities. Efforts to ensure equity and transparency in start-up packages, resources, mentors, sponsors, compensation and leadership are fundamental.

\section{Diversity 4.0: research mandate}

As organizations commit to advancing DEIB, they must engage in funding, researching and disseminating effective strategies to identify bias, describe its prevalence and effect, and outline ways of effectively addressing topics ranging from differences in letters of recommendation to disparities in NIH grant funding. Interventional trials must be designed to test structures and policies to protect against bias and advance DEIB. Data-driven interventions for explicit and implicit biases, such as workshops and short-term interventions on bias literacy, must be studied to mitigate bias. Research must accompany implementation and structured implementation training on how to make informed choices and advance DEIB.

Robust scientific evidence will guide comprehensive, sustainable strategies to eliminate bias, discrimination, harassment and inequities to advance high levels of DEIB. To achieve equity in the cardiovascular workforce and health-care delivery, DEIB must be explicitly addressed, funded, prioritized and quantified over time at the personal, local and health-care system levels.
Only through establishing equity in the health-care workforce can we achieve global health-care equity.

1. Nivet, M. A. Commentary: Diversity 3.0: a necessary systems upgrade. Acad. Med. 86, 1487-1489 (2011)

2. National Science Foundation (NSF). Women, Minorities, and Persons with Disabilities in Science and Engineering https://ncses.nsf.gov/ pubs/nsf19304/ (2019).

3. AIShebli, B. K., Rahwan, T. \& Woon, W. L. The preeminence of ethnic diversity in scientific collaboration. Nat. Commun. 9, 5163 (2018).

4. Burgess, S. et al. Gender equity within medical specialties of Australia and New Zealand: cardiology's outlier status. Intern. Med. J. 50, 412-419 (2020)

5. Khan, M. S. et al. Women training in cardiology and its subspecialties in the United States: a decade of little progress in representation. Circulation 141, 609-611 (2020).

6. Shah, R. U. The $\$ 2.5$ million wage gap in cardiology. JAMA Cardiol. 3, 674-676 (2018).

7. Hall, N. The Kardashian index: a measure of discrepant social media profile for scientists. Genome Biol. 15, 424 (2014).

8. Lewis, S. J et al. Changes in the professional lives of cardiologists over 2 decades. J. Am. Coll. Cardiol. 69, 452-462 (2017).

9. Youngclaus, J. \& Roskovensky, L. An Updated Look at the Economic Diversity of U.S. Medical Students https://www. aamc.org/system/files/ reports/1/october2018anupdatedlookattheeconomicdiversityofu. s. medicalstud. pdf (2018).

10. Carnes, M. et al. Effect of an intervention to break the gender bias habit for faculty at one institution: a cluster randomized, controlled trial. Acad. Med. 90, 221-230 (2015).

\section{Acknowledgements}

R.B.S. has received funding from the European Research Council (ERC) under the European Union's Horizon 2020 research and innovation programme under grant agreement No. 648131 and No. 847770 (AFFECT-EU), and from the German Center for Cardiovascular Research (DZHK e.V.) (81Z1710103), the German Ministry of Research and Education (BMBF 01ZX1408A) and ERACoSysMed3 (031 L0239). E.J.B. receives funding from the NIH (2U54HL1 20163; R01 HL092577; R01 HL141434; R01AG066010; 1R01AG066914) and the AHA (18SFRN34110082).

\section{Competing interests}

R.B.S. has received lecture fees from BMS and Pfizer. E.J.B. declares no competing interests. 